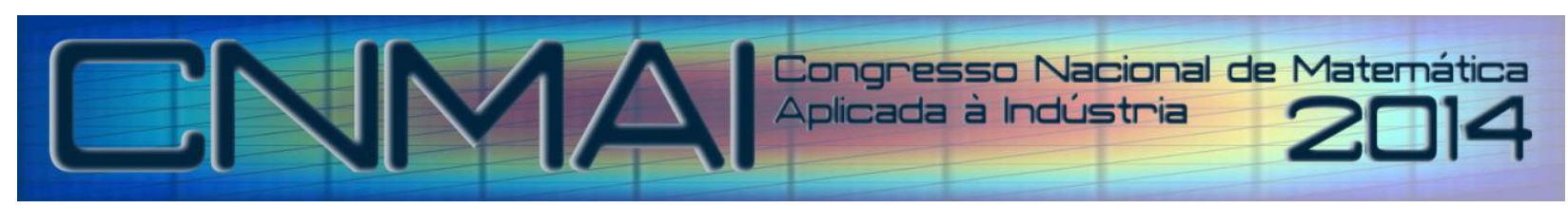

18 a 21 de novembro de 2014, Caldas Novas - Goiás

\title{
MODELO DE TRANSFORMADOR MONOFÁSICO UTILIZANDO UM MODELO GLOBAL PARA PERDAS POR HISTERESE E POR CORRENTES DE EDDY
}

\author{
Rommel Pinheiro França, rommelpfranca@ gmail.com ${ }^{1}$ \\ Niraldo Roberto Ferreira, niraldorferreira@gmail.com ${ }^{2}$ \\ Luiz Alberto Luz de Almeida, lalberto2008@ gmail.com ${ }^{3}$ \\ ${ }^{1}$ Universidade Federal da Bahia - Departamento de Engenharia Elétrica - Escola Politécnica, Rua Aristides Novis, 02, \\ Federação, Salvador - BA - Brasil. CEP 40210-630. \\ ${ }^{2}$ Universidade Federal da Bahia - Departamento de Engenharia Elétrica - Escola Politécnica, Rua Aristides Novis, 02 , \\ Federação, Salvador - BA - Brasil. CEP 40210-630. \\ ${ }^{3}$ Universidade Federal do ABC - Centro de Engenharia, Modelagem e Ciências Sociais Aplicadas. Av. dos Estados, \\ 5001. Bloco A, Torre 1, Sala 723. Bangu. Santo André - SP - Brasil. CEP 09210580.
}

CNMAI2014-0057

Resumo: O sistema elétrico é composto por diversos elementos. Da geração ao consumo, existem equipamentos, com suas especificidades, que podem possuir características lineares ou não. Um exemplo básico de equipamento não linear é o transformador. Essa característica se deve principalmente ao fato deste equipamento possuir bobinas enroladas em núcleos ferromagnéticos, os quais tendem a saturar com o aumento do fluxo magnético. Um sistema elétrico de potência ideal opera com tensões equilibradas. Neste caso, as cargas ligadas a esse sistema devem ser também equilibradas. No entanto, devido à complexidade e às interconexões dos sistemas elétricos, geralmente considera-se que o sistema real opera com tensões entre fases desequilibradas. Além disso, a existência de elementos não lineares no sistema elétrico agrega complexidade aos estudos de comportamento desses sistemas quando da ocorrência de distúrbios. Não obstante, outros fatores contribuem para gerar distúrbios ao sistema elétrico como, por exemplo, os fenômenos eletromagnéticos que podem surgir de descargas atmosféricas, operações de chaveamento (Hendrickson et al., 1953), cargas não lineares e curtos-circuitos. A ferrorressonância, por exemplo, pode surgir em topologias específicas de redes fracamente amortecidas, quando há interação entre as capacitâncias e as reatâncias saturáveis do sistema (Roy and Roy, 2009). Este fenômeno pode provocar sobreaquecimento dos condutores, inclusive das bobinas dos transformadores, além de provocar estresse na isolação com riscos de falhas operacionais e danos aos equipamentos. Dessa forma, para garantir a confiabilidade do sistema, a manutenção deve ser baseada na prevenção e predição. Para isso, modelos matemáticos são desenvolvidos na expectativa de representarem o comportamento físico dos elementos do circuito. Este trabalho pretende apresentar um modelo de transformador monofásico com núcleo saturável do tipo envolvido considerando um modelo algébrico que englobe as perdas por histerese magnética e as perdas por correntes de eddy. Para isso, serão utilizados recursos do software Matlab/Simulink® para implementação do modelo, otimização do processo, análise, comparação e discussão dos resultados.

Palavras-chave: Modelo de transformador monofásico , modelo de histerese $L^{2} P$, núcleo ferromagnético, corrente de inrush.

\section{INTRODUÇÃ̃O}

O transformador é um dos elementos do sistema de potência de maior relevância, motivo pelo qual diversos pesquisadores tem desenvolvido estudos acerca do seu comportamento (Swift, 1971; Rezaei-Zare, 2010; Moses et al., 2010). A maioria dos modelos de transformador considera a histerese magnética. Este fenômeno físico tem sido tratado sob diferentes abordagens, e os modelos baseiam-se principalmente nas teorias de (Preisach, 1935) e de (Jiles and Atherton, 1983). Esses modelos, apesar de apresentarem boa precisão, são relativamente complexos ao possuírem 
equações diferenciais. Neste trabalho, será utilizado um modelo algébrico de histerese (de Almeida et al., 2003) como base para representação do comportamento não linear de um transformador. Optou-se por utilizar um modelo mais simples e que não possuísse equações diferenciais. Serão propostas algumas modificações para este modelo para que o mesmo seja suficiente para a representação do comportamento não linear de um transformador monofásico do tipo núcleo envolvido. Pretende-se, assim, incorporar as perdas por histerese e por correntes parasitas em um único modelo simplificado, baseado em iterações.

\section{DESCRIÇÃO DO MODELO DO TRANSFORMADOR}

\subsection{Equações do circuito elétrico}

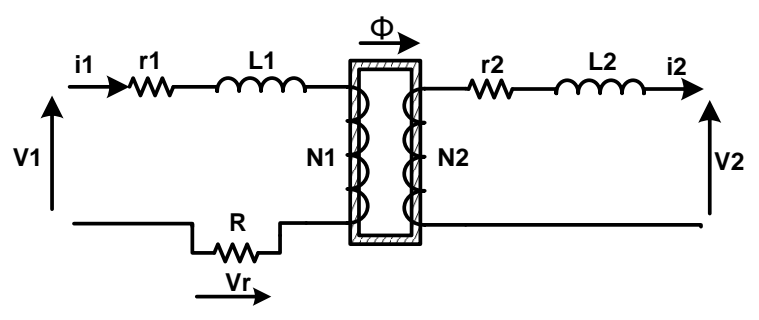

Figura 1. Circuito simplificado de um transformador monofásico.

No transformador de núcleo envolvido da Fig.1, r1, L1, N1, i1, V1 representam a resistência, a indutância, o número de espiras, a corrente e a tensão primárias, respectivamente, e r2, L2, N2, i2 e V2 as grandezas secundárias. Neste circuito, nota-se ainda a resistência R que, neste trabalho, será considerada para a medição da corrente primária. O fluxo magnético $\Phi$ será obtido considerando-se as não linearidades do núcleo magnético representado por um modelo de histerese. Deste circuito, observa-se a equação matricial abaixo:

$$
\left(\begin{array}{r}
\mathrm{v}_{1} \\
-\mathrm{v}_{2}
\end{array}\right)=\left(\begin{array}{cc}
\left(\mathrm{r}_{1}+\mathrm{R}\right) & 0 \\
0 & \mathrm{r}_{2}
\end{array}\right)\left(\begin{array}{l}
\mathrm{i}_{1} \\
\mathrm{i}_{2}
\end{array}\right)+\left(\begin{array}{cc}
\mathrm{L}_{1} & 0 \\
0 & \mathrm{~L}_{2}
\end{array}\right) \frac{\mathrm{d}}{\mathrm{dt}}\left(\begin{array}{l}
\mathrm{i}_{1} \\
\mathrm{i}_{2}
\end{array}\right)+\frac{\mathrm{d}}{\mathrm{dt}}\left(\begin{array}{l}
\Psi_{1} \\
\psi_{2}
\end{array}\right)
$$

\subsection{Equações do circuito magnético}

Baseando-se em (Theocharis et al., 2008), e considerando-se o modelo representado na Fig. 1, obtém-se as seguintes equações:

$$
\begin{aligned}
& \left(\begin{array}{l}
\psi_{1} \\
\psi_{2}
\end{array}\right)=\left(\begin{array}{c}
\mathrm{N}_{1} \\
-\mathrm{N}_{2}
\end{array}\right) \Phi
\end{aligned}
$$

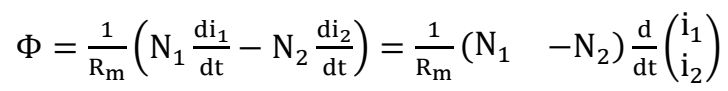

$$
\begin{aligned}
& \frac{\mathrm{d}}{\mathrm{dt}}(\Phi)=\frac{1}{\mathrm{R}_{\mathrm{md}}}\left(\begin{array}{ll}
\mathrm{N}_{1} & -\mathrm{N}_{2}
\end{array}\right) \frac{\mathrm{d}}{\mathrm{dt}}\left(\begin{array}{l}
\mathrm{i}_{1} \\
\mathrm{i}_{2}
\end{array}\right)
\end{aligned}
$$

As equações do circuito elétrico podem ser escritas na forma matricial conforme a Eq. (5).

$$
\begin{aligned}
& \left(\begin{array}{c}
v_{1} \\
-v_{2}
\end{array}\right)=\left(\begin{array}{cc}
\left(r_{1}+R\right) & 0 \\
0 & r_{2}
\end{array}\right)\left(\begin{array}{l}
i_{1} \\
i_{2}
\end{array}\right)+\left(\begin{array}{cc}
L_{1} & 0 \\
0 & L_{2}
\end{array}\right) \frac{d}{d t}\left(\begin{array}{l}
i_{1} \\
i_{2}
\end{array}\right)+\frac{d}{d t}\left(\begin{array}{l}
\psi_{1} \\
\psi_{2}
\end{array}\right) \\
& \left(\begin{array}{l}
\psi_{1} \\
\psi_{2}
\end{array}\right)=\left(\begin{array}{c}
N_{1} \\
-N_{2}
\end{array}\right) \Phi \\
& \Phi=\frac{1}{\mathrm{R}_{\mathrm{m}}}\left(\mathrm{N}_{1} \frac{\mathrm{di}_{1}}{\mathrm{dt}}-\mathrm{N}_{2} \frac{\mathrm{di}_{2}}{\mathrm{dt}}\right)=\frac{1}{\mathrm{R}_{\mathrm{m}}}\left(\begin{array}{ll}
N_{1} & -N_{2}
\end{array}\right) \frac{d}{d t}\left(\begin{array}{l}
i_{1} \\
i_{2}
\end{array}\right) \\
& \frac{d}{d t}(\Phi)=\frac{1}{\mathrm{R}_{\mathrm{md}}}\left(\begin{array}{ll}
N_{1} & -N_{2}
\end{array}\right) \frac{d}{d t}\left(\begin{array}{l}
i_{1} \\
i_{2}
\end{array}\right) \\
& \mathrm{R}_{\mathrm{md}}=\frac{l c}{\mu d \cdot A c} \\
& \mu_{\mathrm{d}}=\frac{d B}{d H}
\end{aligned}
$$


onde,

$\psi$ é o fluxo concatenado

$\mathrm{R}_{\mathrm{m}}$ é a relutância

$l_{c}$ é o comprimento do percurso magnético

$\mathrm{A}_{\mathrm{c}}$ é a área da seção transversal do núcleo magnético

$\mathrm{R}_{\mathrm{md}}$ é a relutância dinâmica

$\mu_{\mathrm{d}}$ a permeabilidade magnética dinâmica

A permeabilidade magnética dinâmica será utilizada no modelo para incorporar os efeitos da histerese ferromagnética. Ademais, neste artigo, o modelo de histerese apresentado considera também as perdas por correntes parasitas, de forma indireta ou simplificada, a partir da consideração da corrente primária total.

\subsection{Aquisição de dados experimentais}

A Fig. 2, a Tab. 1 e a Tab. 2 apresentam as características do núcleo do transformador utilizado. Foram realizados ensaios aplicando-se tensão primária $110 \mathrm{~V}$ e utilizando-se uma bobina oposta como o secundário. Os ensaios consistiram na aquisição de dados experimentais de tensão secundária e corrente primária, durante a energização do transformador ou em regime permanente. A corrente primária é obtida a partir da resistência $R$, de valor conhecido, indicada na Fig. 1.

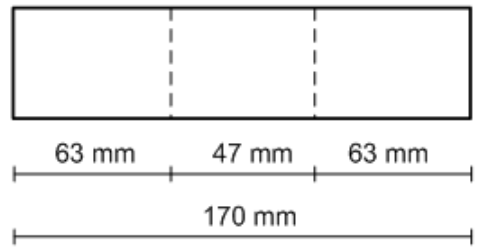

(a)
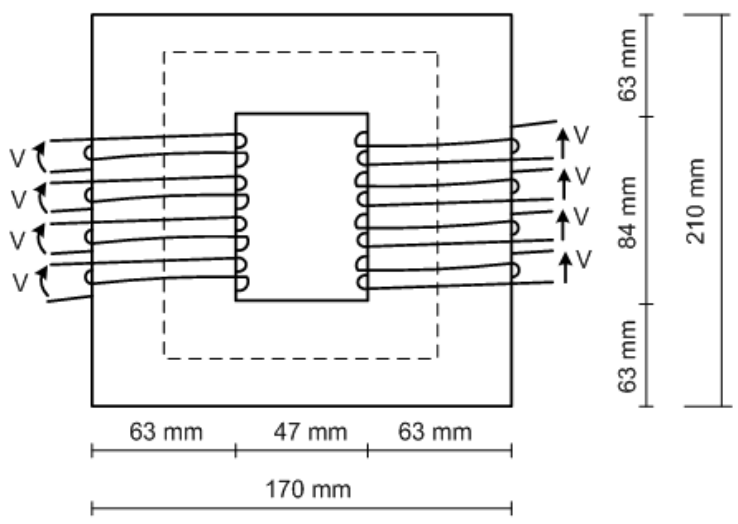

(b)

Figura 2. Dimensões do núcleo do transformador monofásico utilizado. Vista frontal (a) e vista superior (b).

Tabela 1. Dados do Transformador.

\begin{tabular}{|c|c|}
\hline Fabricante & $\begin{array}{c}\text { Equacional Elétrica e } \\
\text { Mecânica LTDA }\end{array}$ \\
\hline Potência & $1 \mathrm{kVA}$ \\
\hline Tensão nominal primária & $110 / 220 \mathrm{~V}$ \\
\hline Tensão nominal secundária & $110 / 220 /$ \\
& $330 / 440 \mathrm{~V}$ \\
\hline Corrente nominal primária & $9,1 / 4,6 \mathrm{~A}$ \\
\hline Corrente nominal secundária & $2,3 \mathrm{~A}(\mathrm{em} 440 \mathrm{~V})$ \\
\hline Resistência por bobina & $1,9 \Omega$ \\
\hline Reatância por bobina & $8,4 \Omega$ \\
\hline Perda aproximada no ferro & $40 \mathrm{~W}$ \\
\hline Perda aproximada no cobre & $80 \mathrm{~W}$ \\
\hline Material do núcleo & Aço-silício \\
\hline
\end{tabular}

Tabela 2. Grandezas medidas ou calculadas.

\begin{tabular}{|c|c|}
\hline Indutância primária (L1) & $0,0233 \mathrm{H}$ \\
\hline Indutância secundária (L2) & $0,0233 \mathrm{H}$ \\
\hline Número de espiras da bobina primária (N1) & 126 \\
\hline Número de espiras da bobina secundária (N2) & 126 \\
\hline Área da seção transversal do núcleo magnético (Ac) & $0,004 \mathrm{~m}^{2}$ \\
\hline
\end{tabular}




\section{Resistência para medição da corrente primária (R) $\quad 10 \Omega$}

A Fig. 3 mostra a curva de histerese para as duas situações: o ensaio de energização do transformador sem carga; e para o regime permanente. Observa-se a ocorrência do processo de acomodação (Torre, 1994) para o inrush, seguindo o estado estacionário.

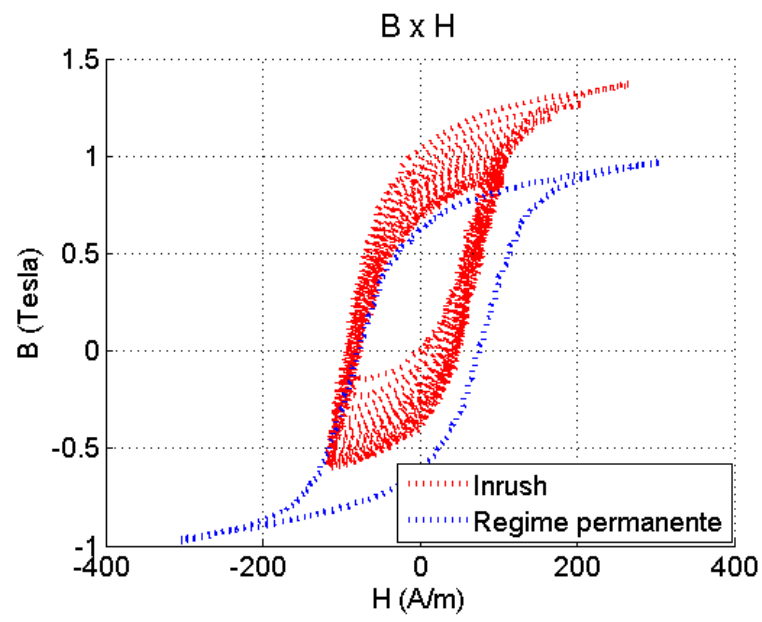

Figura 3. Dados experimentais para curva de histerese durante inrush e para o regime permanente.

\subsection{Modelo de histerese $L^{2} P$}

O modelo de histerese de proximidade ao laço principal (de Almeida et al., 2003) possui apenas quatro parâmetros e reproduz bem os laços internos. Por ser um modelo algébrico, possui baixa complexidade matemática e facilidade de parametrização. O laço principal é representado pela Eq. (11) enquanto que os laços menores, ou ainda, o processo completo, são reproduzidos através da Eq. (12).

$$
\begin{gathered}
\mathrm{M}(\mathrm{H})=\frac{2 \mathrm{M}_{\mathrm{S}}}{\pi} \arctan \left(\frac{\mathrm{H}-\delta \mathrm{H}_{\mathrm{C}}}{\mathrm{h}_{0}}\right) \\
\mathrm{M}(\mathrm{H})=\frac{2 M_{S}}{\pi} \arctan \left(\frac{\mathrm{H}_{\mathrm{pr}} \mathrm{P}\left(\frac{\mathrm{H}-\mathrm{H}_{\mathrm{p}}}{\mathrm{H}_{\mathrm{pr}}}\right)+\mathrm{H}-\delta \mathrm{H}_{\mathrm{C}}}{\mathrm{h}_{0}}\right) \\
\mathrm{H}_{\mathrm{pr}}=\mathrm{h}_{0} \tan \left(\frac{\pi \mathrm{B}_{\mathrm{r}}}{2 \mathrm{~B}_{\mathrm{s}}}\right)+\delta \mathrm{H}_{\mathrm{c}}-\mathrm{H}_{\mathrm{r}}
\end{gathered}
$$

sendo,

$\delta \quad$ sinal da derivada de $\mathrm{H}$

$H \quad$ campo magnético

$H_{C} \quad$ campo coercitivo

$B_{S} \quad$ densidade de fluxo magnético de saturação

$h_{0} \quad$ constante dependente do material

$H_{p} \quad$ campo de proximidade

$H_{p r} \quad$ campo de proximidade em $(\mathrm{Hr}, \mathrm{Br})$

(Hr, Br) ponto de reversão

$\mathrm{P}\left(\frac{\mathrm{H}-\mathrm{H}_{\mathrm{p}}}{\mathrm{H}_{\mathrm{pr}}}\right)$ função de proximidade, definida por:

$$
P(x)=\left\{\begin{array}{ll}
1-\sin (\xi x), & \xi x<\frac{\pi}{2} \\
0 \quad & \xi x>\frac{\pi}{2}
\end{array}, \text { em que } \xi\right. \text { é uma constante arbitrária. }
$$

O ajuste do modelo resume-se em um problema de otimização cujo objetivo consiste em minimizar a função erro médio quadrático (15). Neste trabalho, optou-se por utilizar um método de busca de mínimos globais baseado na teoria simulated annealing (Kirkpatrick et al., 1983). Um procedimento para estimação dos parâmetros deste modelo pode ser visto em (de Almeida et al., 2003). 


$$
J\left(h_{0}, \xi\right)=\sum_{H=H_{\text {sat }}^{-}}^{H=H^{+}}\left\{\begin{array}{l}
{\left[M_{\delta=-1}(H)-M_{\delta=-1}^{\text {experimental }}(H)\right]^{2}} \\
{\left[M_{\delta=+1}(H)-M_{\delta=+1}^{\text {experimental }}(H)\right]^{2}}
\end{array}\right\}
$$

\subsection{Modelo de histerese $L^{2} P \beta$}

A seção anterior apresentou um problema de ajuste do modelo $\mathrm{L}^{2} \mathrm{P}$ aos dados experimentais, decorrente da utilização da corrente primária total, desconsiderando-se um modelo independente para as correntes parasitas. Além disso, o modelo L²P considera apenas a componente irreversível da magnetização. Nesta seção, propõe-se acrescentar a magnetização reversível ao modelo de histerese L²P. Para isso, será considerado o modelo de histerese de Duhem definido na forma (Visitin, 1994):

$$
\frac{d y}{d t}=f_{1}(x, y) \frac{d y^{+}}{d t}-f_{1}(x, y) \frac{d y^{-}}{d t}
$$

cujas funções $f_{1}(x, y)$ e $f_{2}(x, y)$ são arbitrárias, e

$$
\begin{aligned}
& \frac{\mathrm{dx}}{\mathrm{dt}}^{+}=\frac{\left|\frac{\mathrm{dx}}{\mathrm{dt}}\right|+\frac{\mathrm{dx}}{\mathrm{dt}}}{2} \\
& \frac{\mathrm{dx}}{\mathrm{dt}}^{-}=\frac{\left|\frac{\mathrm{dx}}{\mathrm{dt}}\right|-\frac{\mathrm{dx}}{\mathrm{dt}}}{2}
\end{aligned}
$$

A Eq. (16) é também chamada de equação independente da taxa. A estrutura de modelo da Eq. (18) produz duas situações possíveis:

$$
\begin{aligned}
& \frac{d x}{d t}>0 \Rightarrow \frac{d y}{d t}=f_{1}(x, y) \frac{d x}{d t} \\
& \frac{d x}{d t}<0 \Rightarrow \frac{d y}{d t}=f_{2}(x, y) \frac{d x}{d t}
\end{aligned}
$$

Uma representação alternativa para a Eq. (16) pode ser obtida fazendo-se

$$
\frac{d y}{d x}=f(x, y, \delta)
$$

sendo $\delta=\operatorname{sgn}(\dot{\mathrm{x}})$. O operador de histerese definido pela Eq. (16), e colocado na forma da Eq. (21), é chamado de operador de Duhem, denotado por

$$
\mathrm{y}=\mathrm{D}[\mathrm{x}]
$$

O operador de Duhem é apresentado na forma de uma estrutura matemática geral, que define uma classe de modelos. Entretanto, não há metodologia disponível que permita determinar as funções $\mathrm{f}_{1}(\mathrm{x}, \mathrm{y})$ e $\mathrm{f}_{2}(\mathrm{x}, \mathrm{y})$ para um fenômeno de histerese específico. Isto se deve ao fato de que cada aplicação requer uma estrutura de modelo bastante peculiar, e a proposta original de Duhem não estabelece métodos para o desenvolvimento destas estruturas. Em (JilesAtherton, 1983) foi proposto um modelo para a histerese ferromagnética em que o campo externo provoca o crescimento de um certo domínio em detrimento de outros. Jiles postulou que o processo de magnetização é o resultado médio destas interações, conectando as irregularidades do movimento de domínios com a perda de energia no processo de magnetização. O modelo é baseado na hipótese de que a magnetização $\mathrm{M}$ é formada de uma componente irreversível $\mathrm{M}_{\text {irr }}$ e uma componente reversível $\mathrm{M}_{\text {rev }}$, diretamente relacionada com a curva de Langevin

$$
\mathrm{M}_{\mathrm{rev}}=\mathrm{M}_{\mathrm{S}}\left[\operatorname{coth}\left(\frac{\mathrm{H}+\alpha_{\mathrm{L}} \mathrm{M}}{\alpha}\right)-\frac{\alpha}{\mathrm{H}+\alpha_{\mathrm{L}} \mathrm{M}}\right]
$$

sendo $M_{S}$ a magnetização de saturação, $\alpha$ e $\alpha_{L}$ constantes que dependem do material e $H$ o campo magnético aplicado. Jiles considerou que a proporção entre $\mathrm{M}_{\text {rev }}$ e $\mathrm{M}_{\text {irr }}$ é constante para qualquer valor no plano de fase $\mathrm{H}-\mathrm{M}$, e a magnetização total é dada por

$$
\mathrm{M}=(1-\mathrm{c}) \mathrm{M}_{\mathrm{irr}}+\mathrm{c} \mathrm{M}_{\mathrm{rev}}
$$

cuja proporção $\mathrm{c}$ dependente do material pertence ao intervalo $0<c<1$. No modelo de Jiles a componente irreversível $M_{i r r}$ é definida através de um operador de histerese do tipo Duhem denotado por $M_{i r r}=D_{J}[H]$, que resulta em uma magnetização total dada por: 


$$
\mathrm{M}=(1-\mathrm{c}) \mathrm{D}_{\mathrm{J}}[\mathrm{H}]+\mathrm{cM}_{\mathrm{rev}}
$$

De maneira similar ao modelo de Jiles, o modelo L2P pode ser também classificado como um operador de histerese do tipo Duhem, denotado de $\mathrm{D}_{\mathrm{L}^{2} \mathrm{P}}[\mathrm{H}]$. Dessa forma, objetiva-se uma estrutura diferente da proposta por Jiles para a representação das componentes de magnetização. Neste caso, propõe-se que a componente reversível da magnetização seja $\mathrm{M}_{\mathrm{rev}}=\alpha \mathrm{H}$, e a magnetização total definida como

$$
M=M_{i r r}+M_{r e v}=(1-c) D_{L^{2} P}[H]+c . \alpha H
$$

Sendo assim, o modelo $\mathrm{L}^{2} \mathrm{P} \beta$ pode ser definido por:

$$
\mathrm{B}(\mathrm{H})=(1-\mathrm{c}) \frac{2 \mathrm{~B}_{\mathrm{S}}}{\pi} \arctan \left(\frac{\mathrm{H}-\delta \mathrm{H}_{\mathrm{C}}}{\mathrm{h}_{0}}\right)+\mathrm{c} \cdot \alpha \cdot \mathrm{H}
$$

A incorporação da magnetização reversível ao modelo, através da inclusão de mais uma variável, melhora consideravelmente o ajuste do modelo aos dados experimentais, porém sem um aumento expressivo da carga computacional. A estimação do novo parâmetro é feita de forma semelhante seguindo um problema de minimização do erro médio quadrático $(\epsilon)$, de acordo com a Eq. (15).

\section{RESULTADOS E DISCUSSÃO}

\subsection{Modelos de histerese: ajuste ao laço principal}

A Fig. 4 apresenta os ajustes ao laço principal da histerese obtidos pelos modelos de histerese $\mathrm{L}^{2} \mathrm{P}$ e $\mathrm{L}^{2} \mathrm{P} \beta$ para o ensaio em regime permanente, após o processo de acomodação. No segundo, o melhor ajuste obtido forneceu quase a metade do erro do primeiro modelo, garantindo maior precisão.

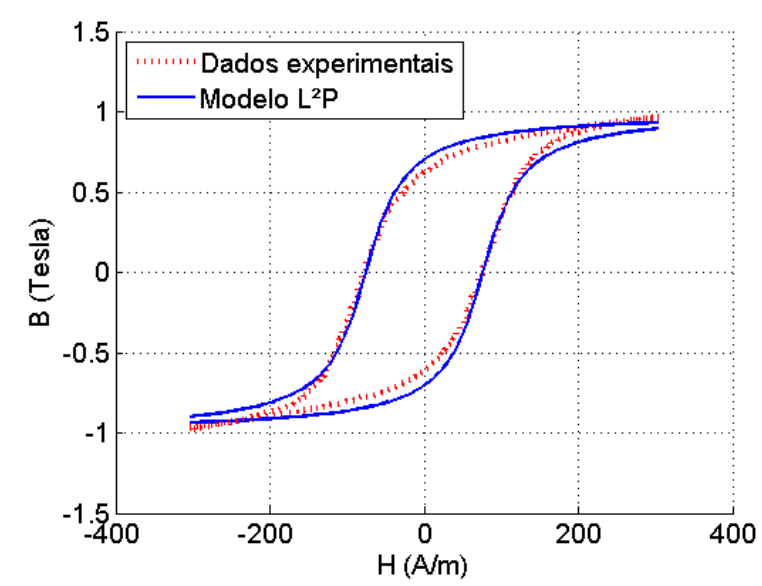

(a)

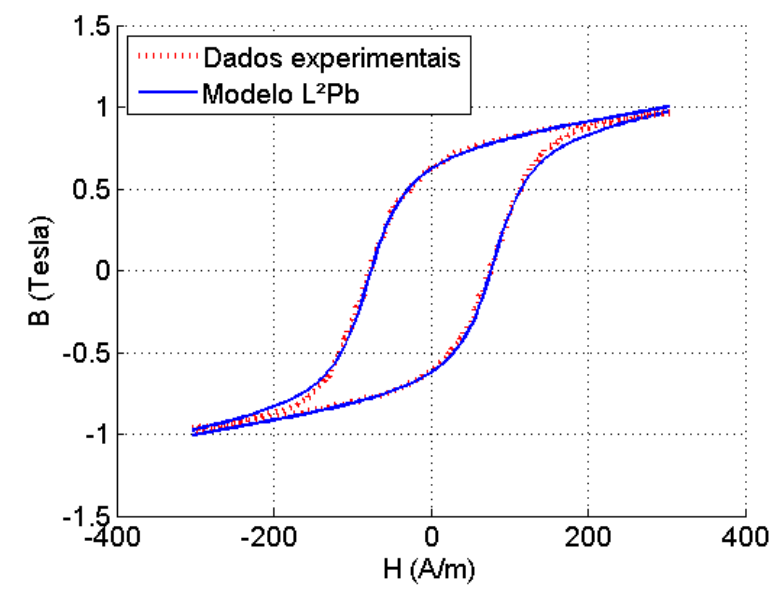

(b)

Figura 4. Ajuste ao laço principal da curva de histerese $B(H)$ pelo modelo.

(a) Modelo $L^{2} P$. Parâmetros: $H_{C}=76,7816 ; h_{0}=37,6170 ; B_{S}=0,9978 ; \epsilon=1,1108 *$.

(b) Modelo L'Pß. Parâmetros: $\quad H_{C}=80,5879 ; h_{0}=34,2277 ; B_{S}=0,8335 ; \alpha=7 \times 10^{-4}$; $\epsilon=0,5722 *$. Obtido pela teoria simulated annealing.

\subsection{Ensaios de energização do transformador sem carga}

\subsubsection{Utilização dos parâmetros ótimos obtidos para o laço principal de histerese: modelo $L^{2} P \beta(0)$}

A Fig. 5 mostra que para este caso os parâmetros obtidos para o laço principal, após a acomodação, não se adéqua aos dados experimentais obtidos durante o inrush. Tal fato provoca saturação intensa no núcleo ferromagnético, produzindo uma corrente de partida elevada. 


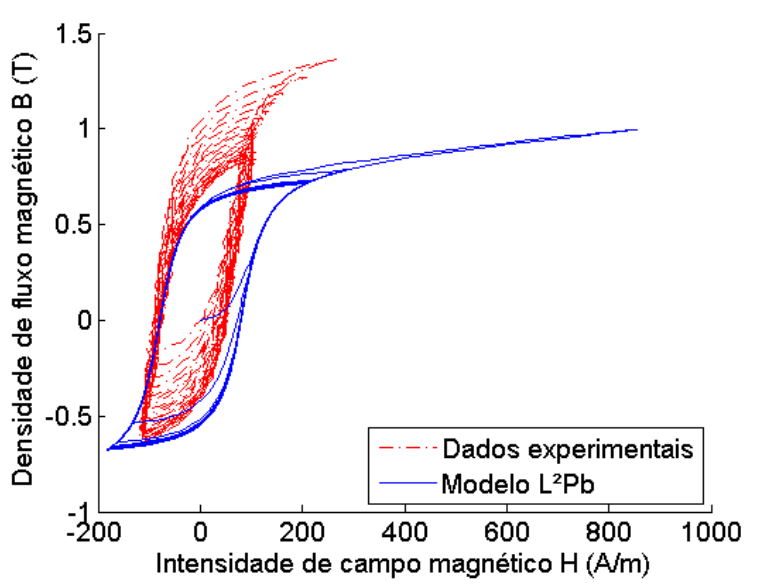

(a)

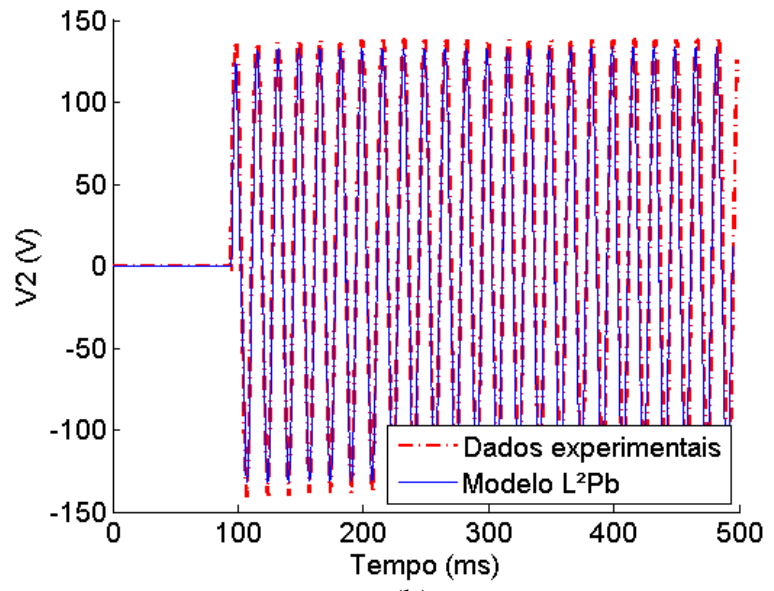

(b)

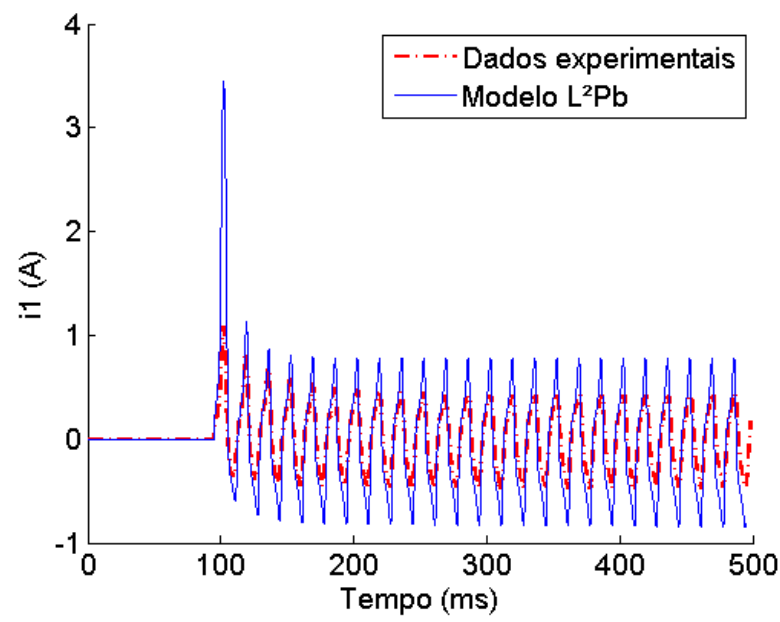

(c)

Figura 5. (a) Relação B(H) com processo de acomodação, (b) tensão secundária e (c) corrente primária. Tensão primária máxima aplicada $138 V$, fase $4^{\circ}$. Parâmetros do modelo $L^{2} P \beta(0): H_{C}=80,5879 ; h_{0}=34,2277$;

$$
B_{S}=0,8335 ; \alpha=7 \times 10^{-4}
$$

\subsubsection{Utilização de parâmetros fixos durante inrush: modelo $L^{2} P \beta(1)$}

Neste caso, foi obtido novo ajuste para os parâmetros do modelo L²P $\beta$, observando-se a ocorrência do processo de acomodação. No entanto, o processo de acomodação experimental segue alterando suas características a cada ciclo, fato que não é possível reproduzir com o modelo L²P $\beta(1)$ a parâmetros fixos, conforme mostra a Fig. 6.

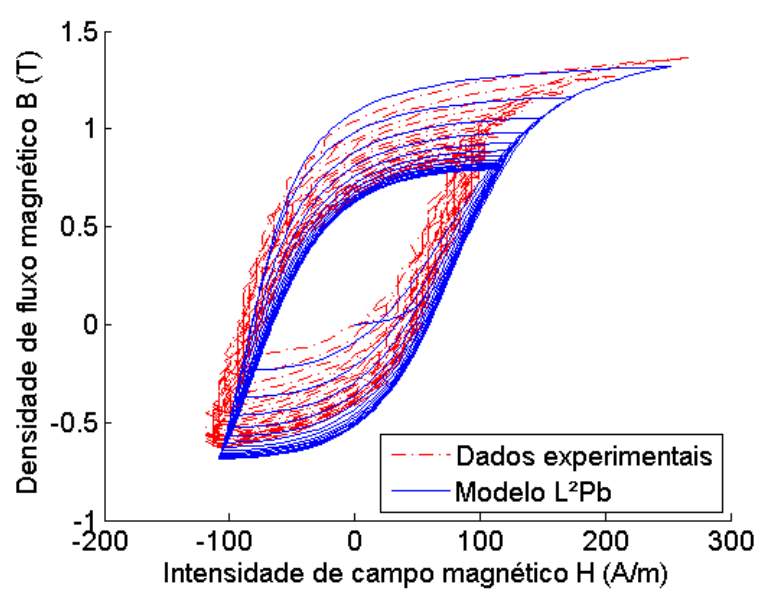

(a)

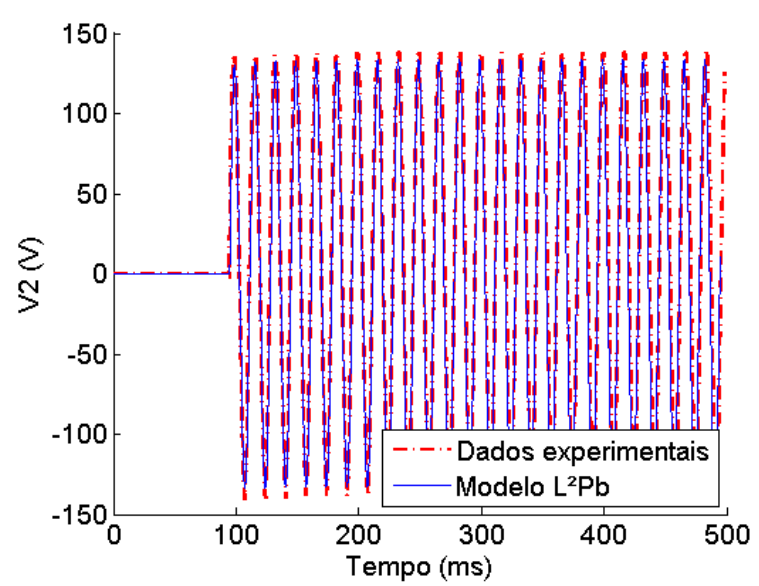

(b) 


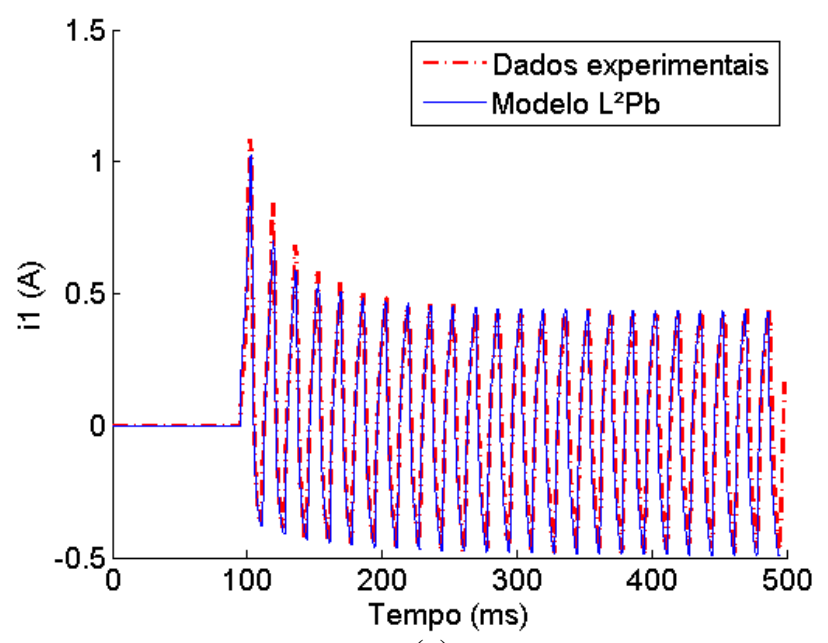

(c)

Figura 6. (a) Relação $B(H)$ com processo de acomodação, (b) tensão secundária e (c) corrente primária. Tensão primária máxima aplicada $138 V$, fase $4^{\circ}$. Parâmetros do modelo $L^{2} P \beta(1): H_{C}=83 ; h_{0}=32,34 ; B_{S}=1,50$; $\xi=0,999 ; \alpha=6,99 \times 10^{-4}$.

3.2.3. Utilização de diferentes parâmetros: comparação entre os modelos $L^{2} P \beta(0)$, parâmetros do laço principal, $L^{2} P \beta(1)$, parâmetros fixos para inrush, e $L^{2} P \beta(2)$, com parâmetros definidos apenas para o primeiro semi-ciclo positivo.

Este caso mostra a utilização de parâmetros diferenciados para a representação do fenômeno da histerese. Observase na Fig. 7 que foi obtido melhor ajuste para o modelo $\mathrm{L}^{2} \mathrm{P} \beta$, configurando, portanto, uma estrutura promissora para a representação das correntes de inrush.

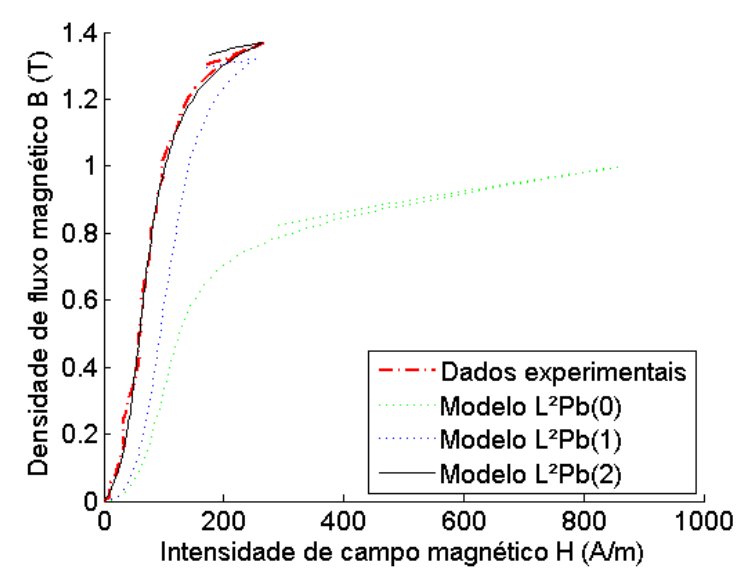

(a)

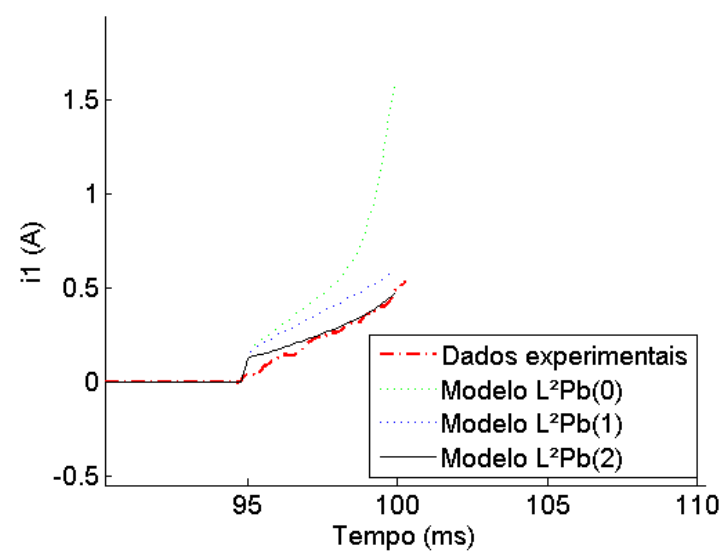

(b)

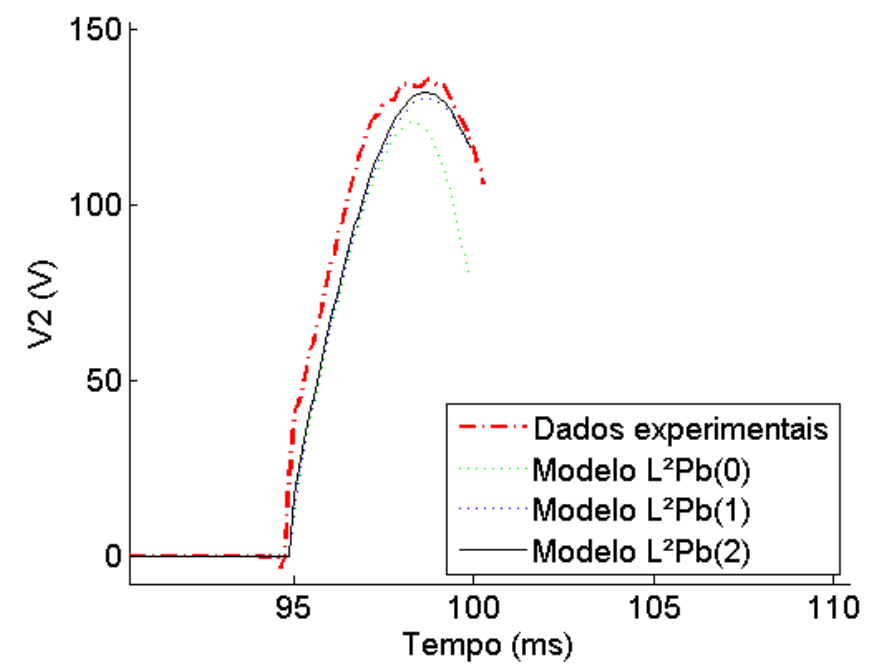

(c) 
Figura 7. (a) Curva de histerese $B(H)$, (b) corrente primária e (c) tensão secundária. Tensão primária máxima aplicada 138V, fase $4^{0}$. Parâmetros do modelo $L^{2} P \beta(1): H_{C}=83 ; h_{0}=32,34 ; B_{S}=1,38 ; \xi=0,999 ; \alpha=$ 6, 99x10 ${ }^{-4}$. Parâmetros do modelo $L^{2} P \beta(2): H_{C}=38,77 ; h_{0}=38,34 ; B_{S}=1,53 ; \xi=0,89999 ; \alpha=$ $0,01 \times 10^{-4}$.

\section{CONCLUSÃO}

$\mathrm{O}$ modelo de histerese $\mathrm{L}^{2} \mathrm{P}$, em sua forma original, considera apenas a magnetização irreversível no material ferromagnético. Este trabalho apresentou uma adaptação ao L²P para considerar também os efeitos da magnetização reversível. Este modelo de histerese adaptado foi inserido como parte de um modelo de transformador monofásico do tipo núcleo envolvido. Resultados foram apresentados comparando-se os dois modelos de histerese para o regime permanente e para a energização do transformador em vazio. Foi possível obter melhores ajustes para a corrente de inrush com a utilização do modelo $\mathrm{L}^{2} \mathrm{P} \beta$, sem, no entanto, necessitar de grandes esforços computacionais.

\section{AGRADECIMENTOS}

À Fundação de Amparo à Pesquisa do Estado da Bahia e à Universidade Federal da Bahia, pela aquisição de equipamentos utilizados nesta investigação.

\section{REFERÊNCIAS}

de Almeida, L. A. L., Deep, G. S., Lima, A. M. N. and Neff, H. 2003. Limiting loop proximity hysteresis model. IEEE Transactions on Magnetics, vol. 39, pp. 523-528.

de Almeida, L. A. L., Deep, G. S., Lima, A. M. N. and Neff, H. 2003. Um modelo diferencial para histerese magnética: representação algébrica recursiva. Revista Controle \& Automação, vol. 14, pp. 58-68.

Hendrickson, P. E; Johnson, I. B; Schultz, N. R. 1953. Abnormal Voltage Conditions Produced by Open Conductors on 3-Phase Circuits Using Shunt Capacitors. AIEE Fall General Meeting, Kansas City, Mo., November 2-6, 1953.

Jiles, D. C. and Atherton, D. L. 1983. Ferromagnetic hysteresis. IEEE Transactions on Magnetics, vol. 19, pp. 21832185.

Kirkpatrick, S; Gelatt, C. D; Vecchi, M. P. 1983. Optimization by Simulated Annealing. Science, New Series, vol. 220, no. 4598, pp. 671-680.

Moses, P. S; Masoum, M. A. S; Toliyat, H. A. 2010. Dynamic Modeling of Three-Phase Asymmetric Power Transformers With Magnetic Hysteresis: No-Load and Inrush Conditions. IEEE Transactions on Energy Conversion, vol. 25, pp. 1040-1047.

Preisach, F. 1935. Über die magnetische Nachwirkung. Zeitschrift für Physik, vol. 94, pp. 277-302.

Rezaei-Zare, A. and Iravani, R. 2010. On the Transformer Core Dynamic During Electromagnetic Transients. IEEE Transactions on Power Delivery, vol. 25, pp. 1606-1619.

Roy, M. and Roy, C. K. 2009. A Study on Ferroresonance and Its Depedence on Instant of Switching Angle of the Source Voltage. Third International Conference on Power Systems, Kharagpur, INDIA December 27-29, paper No:277.

Swift, G. W. 1971. Power Transformer Core Behavior Under Transient Conditions. IEEE Transactions on Power Apparatus and Systems, vol. 90, pp. 2206-2210.

Theocharis, A. D; Milias-Argitis, J; Zacharias, T. 2008. Single-phase transformer model including magnetic hysteresis and eddy currents. Springer-Verlag Elec. Engineering, vol. 90, pp. 229-241, Feb. 2008.

Torre, E. D. 1994. A preisach model for accommodation, IEEE Transactions on Magnetics, No. 5, vol. 30, pp. 27012707.

Visintin, A. 1994. Differential Models of Hysteresis, Springer-Verlag. 


\title{
RESPONSABILIDADE AUTORAL
}

Os autores são os únicos responsáveis pelo conteúdo deste trabalho.

\section{SINGLE-PHASE TRANSFORMER MODEL USING A GLOBAL MODEL FOR HYSTERESIS AND EDDY CURRENTS LOSSES}

\author{
Rommel Pinheiro França, rommelpfranca@ gmail.com ${ }^{1}$ \\ Niraldo Roberto Ferreira, niraldorferreira@ gmail.com ${ }^{2}$ \\ Luiz Alberto Luz de Almeida, lalberto2008@ gmail.com³ \\ ${ }^{1}$ Universidade Federal da Bahia - Departamento de Engenharia Elétrica - Escola Politécnica, Rua Aristides Novis, 02, Federação, \\ Salvador - BA - Brasil. CEP 40210-630. \\ ${ }^{2}$ Universidade Federal da Bahia - Departamento de Engenharia Elétrica - Escola Politécnica, Rua Aristides Novis, 02, Federação, \\ Salvador - BA - Brasil. CEP 40210-630. \\ ${ }^{3}$ Universidade Federal do ABC - Centro de Engenharia, Modelagem e Ciências Sociais Aplicadas. Av. dos Estados, 5001. Bloco A, \\ Torre 1, Sala 723. Bangu. Santo André - SP - Brasil. CEP 09210580.
}

\begin{abstract}
The electrical system is composed of several elements. Generation to consumption, there are equipment with their specificities, which may have linear or non-linear characteristics. A basic example of non-linear equipment is the transformer. This characteristic is due primarily to the fact that this equipment has coils wound on ferromagnetic cores, which tends to saturate with increasing magnetic flux. An ideal electric power system operates with balanced voltages. In this case, the loads connected to the system should be well balanced. However, due to the complexity and interconnections of electrical systems, it is generally considered that the real system operates with unbalanced voltages between phases. Moreover, the existence of non-linear elements in the electrical system adds complexity to the studies of the behavior of these systems in the event of disturbances. Nevertheless, other factors contribute to generate disturbances to the electrical system, for example, the electromagnetic phenomena that can arise from lightning, switching operations (Hendrickson et al., 1953), non-linear loads and short-circuits. The ferroresonance, for example, may arise in specific network topologies weakly damped when there is interaction between the capacitance and saturable reactance in the system (Roy and Roy, 2009). This phenomenon can cause overheating of conductors, including the coils of the transformers, in addition to causing stress in isolation with risks of operational failures and damage to equipment. Thus, to ensure system reliability, maintenance should be based on prevention and prediction. For this, mathematical models are developed in the expectation to represent the physical behavior of the circuit elements. This work aims to present a single-phase core-type transformer model considering an algebraic model encompassing the magnetic hysteresis losses and eddy current losses. For this, resources from Matlab/Simulink® software will be used for model implementation, process optimization, analysis, comparison and discussion of the results.
\end{abstract}

Keywords: Single-phase transformer model, L2P algebraic hysteresis model, ferromagnetic core, inrush current. 